\title{
entre progenies de Cordia trichotoma en una plantación mixta con Dalbergia nigra
}

\section{Genetic divergence in Cordia trichotoma progenies in a multiple cropping system with Dalbergia nigra}

\author{
Wanderley dos Santos ${ }^{*}$, Bruno Marchetti de Souza', Daniele Fernanda Zulian', Gilberto Terra Ribeiro Alves², \\ Mário Luiz Teixeira de Moraes ${ }^{3}$, Valderês Aparecida de Sousa ${ }^{4}$ y Ananda Virginia de Aguiar ${ }^{4}$
}

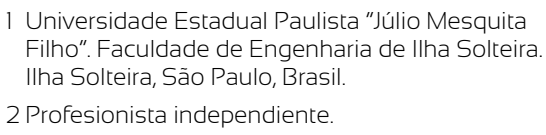

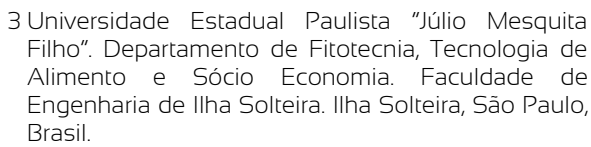

4 Pesquisadora Empresa Brasileira de Pesquisa Agropecuária - EMBRAPA Florestas. Colombo, Paraná, Brasil.

* Autor de correspondencia. wanderley.dossantos@ahotmail.com

\section{RESUMEN}

Cordia trichotoma es una especie nativa que está siendo evaluada para diferentes sistemas forestales. El hecho de que los individuos empleados en las plantaciones posean algún grado de mejoramiento genético tiene muchas ventajas; para lograr este mejoramiento es fundamental la obtención de genotipos superiores, a fin de obtener un mejor rendimiento del producto final. El objetivo del presente estudio fue estimar la divergencia genética de progenies de C. trichotoma en dos diferentes condiciones de textura de suelo, por medio de caracteres fenotípicos. El diseño experimental fue bloques completos al azar, un árbol por parcela con 40 (área 1) y 34 (área 2) repeticiones, con espaciamiento de 3,0 $\mathrm{m} \times 3,0 \mathrm{~m}$. Los caracteres evaluados fueron: diámetro a altura del pecho, altura total de plantas, diámetro de cuello y altura del primer verticilo. Para estimar la divergencia genética y el agrupamiento de Tocher fue utilizado el método REML/BLUP. Las distancias entre las progenies en el área 1 fueron de 2,13 y 47,74 (17 y 15), en el área 2 las distancias entre las progenies fueron de 3,64 (9 y 15) y 48,28 (12 y 7). Las progenies formaron diez y cuatro grupos distintos (área 1 y área 2). Los caracteres que más contribuyeron para divergencia genética fueron diámetro a la altura del pecho-2016 (14,65\%) área 1, y altura total de plantas-2014 $(14,32 \%)$ área 2. Las progenies de C. trichotoma presentaron alta divergencia genética. En programas de mejoramiento, se recomienda el cruzamiento entre progenies de los grupos más divergentes para priorizar la ganancia con selección de los genotipos más productivos.

PALABRAS CLAVE: análisis multivariado, distancia genética, genética cuantitativa, louro-pardo, optimización de Tocher.

\section{ABSTRACT}

Cordia trichotoma is a Brazilian species that has been studied for different forest systems. However, genetic breeding programs could highly improve species use. To obtain a better yield, it is essential to identify the species genotypes with superior performance. Thus, the work objective of this work was to estimate genetic divergence in two $C$. trichotoma progenies tests based on phenotypic traits. The experimental design adopted was the randomized blocks, one plant per plot, using 40 (area one) and 34 (area two) repetitions, using $3.0 \mathrm{~m} \times 3.0 \mathrm{~m}$ spacing. The evaluated traits were diameter at $1.30 \mathrm{~m}$ above the soil $(\mathrm{DBH}-\mathrm{cm})$, total height $(\mathrm{HT}-\mathrm{m})$, collar diameter at $30 \mathrm{~cm}$ above the ground $(\mathrm{DC}-\mathrm{cm})$, and height of the first whorl $(\mathrm{HFW}-\mathrm{cm})$. The estimate of genetic divergence and the Tocher clustering method was performed using the REML / BLUP method. The genetic distances between the progenies in area 1 were 2.13 and 47.74 (17 and 15). In area two the genetic distances between the progenies were 3.64 ( 9 and 15) and 48.28 (12 and 7). The progenies formed 10 and 4 distinct groups, respectively, in area 1 and area 2). The traits that most contributed to genetic divergence were DBH-2016 (14.65\%) in area one, and HT-2014 (14.32\%) in area two. In sumary, the C. trichotoma progenies show high genetic divergence. Regarding breeding programs, it is recommended to cross progenies from the most divergent groups to heighten the genetic gain through the selection of more productive genotypes.

KEYWORDS: multivariate analysis, genetic distance, quantitative genetics, louro-pardo, Tocher optimization. 


\section{INTRODUCCIÓN}

Cordia trichotoma (Vell,) Arrabida ex Steud., popularmente conocida como louro-pardo, es una especie nativa que está siendo evaluada en plantaciones forestales para formar sistemas silvopastoriles, agroforestales y plantaciones comerciales puras. El potencial económico de la especie puede ser comprobado por su desempeño en crecimiento, que puede llegar hasta $35 \mathrm{~m}$ de altura, diámetro a la altura de pecho superior a $100 \mathrm{~cm}$ y fuste de hasta $15 \mathrm{~m}$ de altura (Ministério do Meio Ambiente [MMA], 2011). No hay informaión del uso comercial de la madera, sin embargo, algunos trabajos destacan que puede ser empleada en mueblería, embarcaciones, barriles, marcos, etc (Correa y Gonçalves, 2012). La plantación de C. trichotoma en diversos sistemas de plantación presenta más ventajas cuando los individuos tienen algún grado de mejoramiento genético. Por ello, es fundamental la obtención de genotipos superiores para integrar estos sistemas, a fin de obtener un mejor rendimiento del producto final (Santos et al., 2016).

Para la identificación de individuos superiores es necesario el desarrollo de programas de mejoramiento genético para cada especie. El procedimiento adecuado para iniciar un programa de mejoramiento es a partir de ensayos de procedencias o progenies. De esa manera, redes de ensayos experimentales son establecidas para la caracterización de la diversidad genética de la población de mejora, con la que se dimensiona la diversidad genética de la población probada y sirve como guía para la adopción de estrategias adoptadas (Cruz, 2006). Esta caracterización es extremadamente importante para una selección adecuada de los individuos superiores. De esta forma, se garantizan la diversidad existente en una población, el progreso genético y la obtención de genotipos productivos (Cui, Thomas, Carter, Joseph y Randy, 2001). En un programa de mejoramiento genético, la población base debe presentar variabilidad genética y valores medios de los caracteres productivos de interés económico a ser mejorados (Negreiros, Bergo, Migueloni y Pereira, 2013). La identificación de genotipos superiores con base en la divergencia genética es la estrategia más adecuada para iniciar un programa de mejoramiento (Cruz y Carneiro, 2003). Sin embargo, es importante resaltar que es más efectivo realizar cruzamientos entre genotipos altamente divergentes y que presenten buen potencial productivo (Junior, Lima, Araújo, Lima y Lima, 2014). Posterior a la obtención de la divergencia genética entre los pares de progenies es importante utilizar métodos de aglomeración para separarlos en grupos. El método de optimización de Tocher se constituye en un método de agrupamiento simultáneo y tiene el criterio de presentar la distancia media dentro de los grupos siempre menor que la distancia media entre los grupos (Cruz, Regazzi y Carneiro, 2004, Cruz y Carneiro, 2003).

\section{OBJETIVOS}

Los objetivos del estudio fueron evaluar la divergencia genética entre progenies de Cordia trichotoma en dos diferentes condiciones de textura de suelo, así como identificar las mejores posibilidades de cruzas controladas entre los individuos de las progenies más promisorias en productividad.

\section{MATERIALES Y MÉTODOS}

Semillas provenientes de polinización abierta de 30 árboles matrices de Cordia trichotoma fueron recolectadas en la reserva de la Empresa Vale y en pequeños fragmentos forestales localizados entre los municipios de SooretamaES, Brasil (19²11'22,91"S, 40 5'50,48"W) y Linhares-ES, Brasil (19² 23' 40,71" S, $40^{\circ} 3^{\prime}$ 51,40" W). Las semillas recolectadas fueron germinadas en vivero y plantadas en campo en dos áreas con diferente textura de suelo y en un sistema mixto con progenies de Dalbergia nigra (Vell.) Allemão ex Benth. en junio de 2012 en Sooretama, Espírito Santo, Brasil. El municipio de Sooretama-ES se encuentra en la zona natural de tierras calientes, planas y secas. El clima, de acuerdo con la clasificación de Koppen- Geeiger y Af, es tropical caliente húmedo con lluvias en el verano e invierno seco (Alvares et al., 2013). El índice pluviométrico es de $1200 \mathrm{~mm} /$ año y la temperatura media es $23,4{ }^{\circ} \mathrm{C}$. El relieve es predominantemente plano (Instituto Capixaba de 
Pesquisa, Assistência Técnica y Extensão Rural [Incaper], 2013).

El diseño experimental fue bloques completos al azar, un árbol por parcela con espaciamiento de 3,0 $\mathrm{m} \times 3,0 \mathrm{~m}$ entre las especies y $6,0 \mathrm{~m} \times 6,0 \mathrm{~m}$ entre la misma especie. En el área 1 el suelo es arenoso y se realizaron 40 repeticiones. En el área 2 el suelo es arcilloso y se realizaron 34 repeticiones.

Los árboles de las dos áreas fueron medidos anualmente desde el 2013 (12 meses) hasta 2016 (48 meses), los caracteres evaluados fueron: altura de plantas (ALT; m), altura del primer verticilo (APV; $\mathrm{m}$ ), diámetro de cuello (DC; $\mathrm{cm}$ a $30 \mathrm{~cm}$ del suelo) y diámetro a la altura del pecho (DAP; cm a 1,30 $\mathrm{m}$ del suelo).

Para obtener divergencia genética, se estimaron los componentes de la varianza y los parámetros genéticos (Santos et al., 2020), se obtuvieron mediante el método REML / BLUP (máxima verosimilitud restringida / mejor predicción lineal insesgada), utilizando datos no balanceados y el software genético-estadístico SELEGEN®-REML / BLUP (Resende, 2007). Las pruebas de progenie de polinización abierta (medias hermanas) se realizaron considerando un diseño completo de bloques al azar, una parcela de árbol, un sitio y una sola población.

$$
y=\boldsymbol{X} r+Z a+e
$$

donde

$y=$ vector de datos

$r=$ vector de los efectos de repetición (asumidos como fijos) agregados a la media general

$a=$ vector de efectos genéticos aditivos individuales (asumidos como aleatorios)

$e=$ vector de errores o residuos (aleatorio).

Las letras mayúsculas representan las matrices de incidencia de estos efectos.

La divergencia genética entre las progenies fue estimada por medio de la distancia generalizada de Mahalanobis $\left(\mathrm{D}^{2}\right)$, descrita por la expresión propuesta por Cruz y Carneiro (2003):

$$
D_{i i}^{2}=\delta^{\prime} \Psi^{-1} \delta
$$

donde:

$D_{i i}^{2}=$ distancia de Mahalanobis entre los genotipos i y i'

$\delta^{\prime}=\left[d_{1}, d_{2},,, d_{v}\right]$, siendo $d_{j}=Y_{i j}-Y_{i \prime j}$

$\Psi=$ matriz de varianzas y covarianzas residuales

$Y_{i j}=$ media del $i$-ésimo genotipo en relación con la $j$-ésima variable

Posterior a la obtención de la matriz de distancia $\left(\mathrm{D}^{2}\right)$, fue realizado el análisis de agrupamiento por el método de optimización de Tocher (Cruz y Regazzi, 2001), en el cual se adoptó el criterio de que la media de los valores de $\mathrm{D}^{2}$ (intraclúster) debe ser menor que los valores de $\mathrm{D}^{2}$ (interclúster). La divergencia genética fue obtenida por el método REML/BLUP, utilizándose el software genéticoestadístico SELEGEN ${ }^{\circledR}$-REML/BLUP (Resende, 2016). La contribución de los caracteres para la formación de los grupos fue obtenida con el uso del programa computacional GENES $^{\circledR}$ (Cruz, 2006).

\section{RESULTADOS Y DISCUSIÓN}

La información sobre la divergencia de un individuo candidato contribuye a la planeación futura y utilización eficiente de los recursos genéticos para potencializar y maximizar el crecimiento y rendimiento en la selección (Santos et al., 2016). La distancia máxima observada entre las progenies del área 1 fue de $\mathrm{D}^{2}=47,74$ entre las progenies 17 y 15 y la mínima fue de $\mathrm{D}^{2}=2,13$ entre las progenies 25 y 27. Las progenies en el área 2 presentaron distancia máxima de $\mathrm{D}^{2}=48,28$ entre las progenies 9 y 15 , y la distancia mínima fue de $\mathrm{D}^{2}=3,64$ entre las progenies 12 y 7 (Tabla 1).

Con base en los resultados, pueden ser seleccionados los individuos de las progenies más divergentes y con mayor desempeño productivo y/o mayor valor genotípico para realización de los cruzamientos controlados. Otra información importante está relacionada con la Distancia de Mahalanobis, la cual posibilita cuantificar la contribución relativa de los caracteres para la divergencia genética (Tabla 2). Eso permite conocer cuáles progenies pueden ser 
utilizadas como una matriz de cruzamiento y cuál caracter debe ser priorizado y puede auxiliar en la selección de progenies más promisoras en productividad. Santos et al. (2020) relatan, para este mismo experimento, variación genética entre progenies de 4,72\% a 19,08\% para los caracteres APV-2016 y DC-2014 en el área 1. Entre tanto, en el área 2, varió de 10,98\% a 23,18\% para los caracteres ALT-2016 y APV-2015. Las estimaciones del coeficiente de variación genética individual fueron de $9,45 \%$ a $38,16 \%$ para los caracteres APV-2016 y DC-2014 en el área 1. En el área 2, varió de 21,96\% a 46,36\% para ALT-2016 y DAP2015, respectivamente.

TABLA 1. Distancias de Mahalanobis $\left(\mathrm{D}^{2}\right)$ en las áreas 1 y 2 para el diámetro a la altura del pecho (DAP; $\left.\mathrm{cm}\right)$, altura de planta (ALT; m), diámetro de cuello (DC; $\mathrm{cm})$ y altura del primer verticilo (APV; $\mathrm{cm})$ en progenies de Cordia trichotoma.

\begin{tabular}{|c|c|c|c|c|c|c|c|c|c|}
\hline \multicolumn{10}{|c|}{$D^{2}$ de Mahalanobis } \\
\hline \multicolumn{5}{|c|}{ Distancias Área 1} & \multicolumn{5}{|c|}{ Distancias Área 2} \\
\hline Prog. & Mayor & Prog. & Menor & Prog. & Prog. & Mayor & Prog. & Menor & Prog \\
\hline 23 & 34,71 & 15 & 9,89 & 21 & 8 & 35,33 & 25 & 5,73 & 5 \\
\hline 19 & 39,35 & 15 & 9,01 & 2 & 19 & 41,45 & 16 & 11,38 & 10 \\
\hline 7 & 31,50 & 17 & 5,12 & 2 & 7 & 38,24 & 9 & 3,64 & 12 \\
\hline 24 & 35,81 & 17 & 4,42 & 14 & 27 & 38,24 & 26 & 6,66 & 23 \\
\hline 21 & 29,56 & 17 & 6,65 & 27 & 21 & 37,77 & 9 & 8,70 & 28 \\
\hline 4 & 23,27 & 8 & 4,05 & 16 & 4 & 33,04 & 15 & 4,53 & 14 \\
\hline 27 & 29,59 & 15 & 2,13 & 25 & 11 & 43,39 & 9 & 7,73 & 3 \\
\hline 11 & 39,83 & 17 & 9,17 & 14 & 12 & 32,42 & 25 & 3,64 & 7 \\
\hline 18 & 36,02 & 9 & 10,87 & 7 & 23 & 32,25 & 25 & 5,61 & 10 \\
\hline 12 & 24,57 & 17 & 4,76 & 4 & 15 & 48,28 & 9 & 25,45 & 22 \\
\hline 28 & 46,66 & 20 & 16,65 & 12 & 25 & 39,45 & 15 & 16,38 & 18 \\
\hline 22 & 34,95 & 15 & 4,80 & 24 & 17 & 40,46 & 29 & 13,93 & 20 \\
\hline 8 & 32,74 & 1 & 6,49 & 5 & 13 & 39,92 & 15 & 5,89 & 5 \\
\hline 25 & 27,38 & 15 & 2,13 & 27 & 3 & 35,18 & 25 & 6,12 & 14 \\
\hline 17 & 47,74 & 15 & 13,50 & 16 & 2 & 34,64 & 25 & 5,07 & 7 \\
\hline 13 & 26,03 & 18 & 5,86 & 16 & 26 & 38,24 & 27 & 12,08 & 2 \\
\hline 3 & 36,02 & 9 & 8,46 & 12 & 10 & 28,36 & 25 & 5,61 & 23 \\
\hline 20 & 38,77 & 17 & 13,23 & 27 & 14 & 28,76 & 15 & 4,53 & 4 \\
\hline 2 & 26,10 & 15 & 5,12 & 7 & 6 & 34,21 & 15 & 4,86 & 7 \\
\hline 26 & 33,89 & 20 & 7,25 & 13 & 9 & 48,28 & 15 & 19,98 & 14 \\
\hline 10 & 39,66 & 15 & 8,01 & 22 & 5 & 32,55 & 9 & 5,73 & 8 \\
\hline 14 & 26,16 & 3 & 4,42 & 24 & 16 & 41,45 & 19 & 9,43 & 22 \\
\hline 6 & 33,94 & 15 & 6,64 & 24 & 1 & 39,20 & 25 & 5,76 & 10 \\
\hline 9 & 38,35 & 11 & 10,68 & 21 & 29 & 40,46 & 17 & 9,04 & 6 \\
\hline 5 & 31,66 & 6 & 6,49 & 8 & 30 & 39,71 & 15 & 10,07 & 28 \\
\hline 16 & 24,37 & 19 & 4,05 & 4 & 20 & 40,84 & 15 & 8,01 & 10 \\
\hline 1 & 34,35 & 20 & 9,08 & 7 & 18 & 33,73 & 15 & 7,25 & 5 \\
\hline 29 & 29,76 & 17 & 7,66 & 14 & 28 & 31,93 & 25 & 8,49 & 2 \\
\hline 30 & 35,29 & 20 & 5,61 & 12 & 22 & 28,08 & 25 & 6,75 & 5 \\
\hline \multicolumn{5}{|c|}{ Máximo: 47,74 Progenies: 17 y 15} & \multicolumn{5}{|c|}{ Máximo: 48,28 Progenies: 9 y 15} \\
\hline Mínimo: 2 & ogenies: & y 27 & & & mo: 3,6 & enies: 12 & & & \\
\hline
\end{tabular}


TABLA 2. Contribución relativa de los caracteres a la divergencia en las áreas 1 y 2 para los caracteres: diámetro a altura del pecho (DAP, $\mathrm{cm}$ ), altura de planta (ALT, $\mathrm{m})$, diámetro de cuello $(\mathrm{DC}, \mathrm{cm})$ y altura del primer verticilo (APV, $\mathrm{cm}$ ) en progenies de Cordia trichotoma.

\begin{tabular}{cccc}
\hline & Área 1 & & \multicolumn{2}{c}{ Área 2} \\
\hline Variable & $\%$ & Variable & 11,55 \\
\hline ALT-2013 & 10,35 & ALT-2013 & 10,64 \\
DC-2014 & 7,65 & DC-2014 & 14,32 \\
ALT-2014 & 10,05 & ALT-2014 & 9,36 \\
APV-2014 & 9,77 & APV-2014 & 6,35 \\
DAP-2015 & 7,62 & DAP-2015 & 8,2 \\
ALT-2015 & 11,35 & ALT-2015 & 10,47 \\
APV-2015 & 9,69 & APV-2015 & 9,76 \\
DAP-2016 & 14,65 & DAP-2016 & 11,36 \\
ALT-2016 & 7,36 & ALT-2016 & 7,99 \\
APV-2016 & 11,51 & APV-2016 & \\
\hline
\end{tabular}

El DAP-2016 fue el que más contribuyó a la divergencia $(14,65 \%)$ y el DC-2014 fue el que menos contribuyó (7,65\%), en el área 1. En el área 2, la ALT-2014 fue la que más contribuyó a la divergencia (14,32\%) y el DAP-2015 $(6,35 \%)$ fue el que menos contribuyó a la divergencia. Los demás caracteres también registraron participación funcional en la divergencia de las progenies. Las estimativas de correlaciones genéticas confirmaron asociaciones positivas significativas entre todos los caracteres, excepto para APV-2014 y DAP-2015 en el área 2. El mayor valor fue observado entre los caracteres DAP-2016 y ALT-2015 en las dos áreas (0,82 área 1 y 0,93 área 2). El APV-2015 tiene asociaciones negativas con ALT-2014 (-0,07) y DAP2015 (-0,13) (Santos et al., 2020). Kumar, Ravichandran, Dobhal y Kumar (2016) encontraron que el DAP fue el de mayor contribución $(23,02 \%)$, seguido por DC $(22,54 \%)$ y el diámetro de copa fue el de menor contribución (6,3\%). Singh (1981), basado en $\mathrm{D}^{2}$ de Mahalanobis, considera de menor importancia caracteres que expresan menor variabilidad. Se sugiere, en este caso, que se descarten los caracteres que presentaron la menor contribución a la divergencia como el DC-2014 (7,65\%) en el área 1 y DAP2015 (6,35\%) en el área 2 (Tabla 2). El gran interés en la evaluación de la importancia relativa de los caracteres reside en la posibilidad de descartar caracteres con poca contribución a la discriminación del material evaluado, disminuyendo mano de obra, tiempo y costo invertidos en la experimentación (Alves, García, Cruz y Figueira, 2003; Correa y Gonçalves, 2012). Eso garantizará la elección del caracter más adecuado con la finalidad de mejoramiento. Santos et al. (2020) relataron ganancia genética a partir de DAP a los cuatro años de edad, en ambas áreas, siendo de $5,72 \%$ a $15,45 \%$. La mayor intensidad de selección (2,5\%) mostró pequeña población de tamaño efectivo $(7,15)$ y diversidad genética $(0,09)$ en área 1 y en área $2(=6,57$ y D $=0,08)$.

Informaciones sobre las medias y coeficiente de variación $(\mathrm{CV} \%)$ son base para formación de los grupos y pueden también auxiliar al mejorador, al detectar la divergencia entre grupos (Tabla 3). Los grupos formados por las progenies del área 1 presentaron las medias superiores para todos los caracteres. El grupo tres presentó valores medios más altos para altura $(677,08 \mathrm{~cm})$ y diámetro de copa $(392,71 \mathrm{~cm})$ y mantuvo el mayor valor medio $(187,04)$ sobre los valores medios de todos evaluados en el agrupamiento de Tocher (Tabla 3), lo que indica el desempeño superior de la progenie 33 del grupo 3. 
TABLA 3. Medias generales $(\widehat{m})$, coeficiente de variación $(C V \%)$ intergrupos y agrupamiento de Tocher a partir de las distancias genéticas de Mahalanobis en las áreas 1 y 2 para el diámetro a la altura delpecho (DAP-cm), altura de planta (ALT-m), diámetro del cuello (DC$\mathrm{cm})$ y altura del primer verticilo (APV-cm) en progenies de Cordia trichotoma.

\begin{tabular}{|c|c|c|c|c|c|c|c|c|c|c|c|c|}
\hline Área 1 & & & & & & Mec & lias par & cada & rupo & & & \\
\hline Grupos & Progenies & Total & $\begin{array}{l}A L T \\
2013\end{array}$ & $\begin{array}{c}D C \\
2014\end{array}$ & $\begin{array}{l}A L T \\
2014\end{array}$ & $\begin{array}{l}A P V \\
2014\end{array}$ & $\begin{array}{l}D A P \\
2015\end{array}$ & $\begin{array}{l}A L T \\
2015\end{array}$ & $\begin{array}{l}A P V \\
2015\end{array}$ & $\begin{array}{l}D A P \\
2016\end{array}$ & $\begin{array}{l}A L T \\
2016\end{array}$ & $\begin{array}{l}A P V \\
2016\end{array}$ \\
\hline 1 & $\begin{array}{c}7242142718122282513 \\
226145162930\end{array}$ & 18 & 0,74 & 41,36 & 1,83 & 0,28 & 4,65 & 2,99 & 0,42 & 5,07 & 3,46 & 0,56 \\
\hline 2 & 91 & 2 & 0,70 & 46,44 & 1,79 & 0,32 & 4,55 & 2,61 & 0,49 & 4,95 & 3,04 & 0,60 \\
\hline 3 & 310 & 2 & 0,87 & 41,01 & 1,74 & 0,24 & 4,78 & 2,96 & 0,36 & 5,44 & 3,33 & 0,46 \\
\hline 4 & 2328 & 2 & 0,76 & 40,57 & 1,95 & 0,34 & 4,19 & 3,06 & 0,43 & 5,27 & 3,49 & 0,61 \\
\hline 5 & 11 & 1 & 0,66 & 44,93 & 2,11 & 0,26 & 4,86 & 3,16 & 0,34 & 5,41 & 3,95 & 0,48 \\
\hline 6 & 19 & 1 & 0,81 & 46,06 & 2,07 & 0,31 & 4,41 & 2,96 & 0,52 & 5,15 & 3,65 & 0,68 \\
\hline 7 & 20 & 1 & 0,91 & 46,08 & 2,42 & 0,31 & 5,58 & 3,71 & 0,44 & 5,69 & 4,02 & 0,54 \\
\hline 8 & 6 & 1 & 0,71 & 44,91 & 1,85 & 0,24 & 5,61 & 3,32 & 0,53 & 5,91 & 3,81 & 0,71 \\
\hline 9 & 17 & 1 & 0,89 & 47,05 & 2,02 & 0,29 & 5,12 & 3,16 & 0,42 & 5,15 & 3,36 & 0,79 \\
\hline 10 & 15 & 1 & 0,79 & 54,38 & 2,03 & 0,34 & 5,79 & 3,51 & 0,41 & 6,47 & 4,01 & 0,49 \\
\hline$\widehat{m}$ & & & 0,76 & 42,81 & 1,88 & 0,28 & 4,74 & 3,03 & 0,43 & 5,22 & 3,51 & 0,57 \\
\hline CV (\%) & & & 62,08 & 55,11 & 47,1 & 72,7 & 58,3 & 46,61 & 72,01 & 55,35 & 40,36 & 75,66 \\
\hline Total & & 30 & & & & & & & & & & \\
\hline Área 2 & & & & & & $\mathrm{Mec}$ & lias par & cada $\mathrm{c}$ & rupo & & & \\
\hline Grupos & Progenies & Total & $\begin{array}{l}A L T \\
2013\end{array}$ & $\begin{array}{c}D C \\
2014\end{array}$ & $\begin{array}{l}A L T \\
2014\end{array}$ & $\begin{array}{l}A P V \\
2014\end{array}$ & $\begin{array}{l}\text { DAP } \\
2015\end{array}$ & $\begin{array}{l}A L T \\
2015\end{array}$ & $\begin{array}{l}A P V \\
2015\end{array}$ & $\begin{array}{l}D A P \\
2016\end{array}$ & $\begin{array}{l}A L T \\
2016\end{array}$ & $\begin{array}{l}A P V \\
2016\end{array}$ \\
\hline 1 & $\begin{array}{c}81972721411122317133 \\
226101465161293020 \\
182822\end{array}$ & 27 & 0,77 & 36,33 & 1,87 & 0,31 & 3,53 & 2,54 & 0,52 & 3,92 & 2,99 & 0,55 \\
\hline 2 & 25 & 1 & 0,58 & 38,14 & 1,96 & 0,35 & 3,83 & 2,87 & 0,76 & 4,52 & 4,33 & 0,71 \\
\hline 3 & 9 & 1 & 0,96 & 42,76 & 2,35 & 0,46 & 3,91 & 2,85 & 0,89 & 4,21 & 3,29 & 0,95 \\
\hline 4 & 15 & 1 & 0,82 & 38,82 & 1,78 & 0,31 & 3,71 & 2,33 & 0,32 & 3,86 & 3,87 & 0,42 \\
\hline$\widehat{m}$ & & & 0,77 & 36,68 & 1,89 & 0,32 & 3,55 & 2,55 & 0,53 & 3,94 & 3,06 & 0,56 \\
\hline CV (\%) & & & 60,99 & 57,02 & 48 & 64,99 & 67,4 & 53,81 & 66,78 & 64,71 & 62,78 & 66,01 \\
\hline Total & & 30 & & & & & & & & & & \\
\hline
\end{tabular}

El empleo del método de optimización de Tocher, basado en las distancias de Mahalanobis (D2), posibilitó la formación de 10 grupos (área 1) y cuatro grupos (área 2), con concentración de aproximadamente $60 \%$ y $90 \%$ de las progenies en un único grupo (grupo 1) en las áreas 1 y 2, respectivamente (Tabla 3). Los demás grupos fueron formados por máximo dos progenies para el área 1. Costa et al. (2016) estudiando progenies de Eucalyptus camaldulensis 
Dehnh por el método de Tocher, considerando la distancia de Mahalanobis, obtuvo la formación de 14 grupos diferentes. Donde aproximadamente $86 \%$ de las progenies se agruparon como parte del grupo 1, siendo el grupo más divergente el grupo 14 con una progenie. Coser, Motoike, Corrêa, Pires y Resende (2016), estudiando 52 progenies de Acrocomia aculeata (Jacq.) Lodd. Mart, observaron la formación de cuatro grupos, de los cuales el primer grupo reunió $92 \%$ de las progenies y el grupo 4 apenas una progenie. Kumar et al. (2016) mencionan la formación de siete grupos para clones de Dalbergia sissoo Linn., el grupo 1 compuesto por 25 clones, grupo 2 con cuatro clones, seguido por el grupo 4 con tres clones, y los dos grupos restantes solo tivieron un clon.

Existen diferencias en relación con la formación de grupos en las dos áreas, lo que puede ser resultado de la divergencia entre los individuos de la misma progenie, textura del suelo y efectos del sistema de plantío mixto con Dalbergia nigra. Los suelos de las dos parcelas son muy semejantes en clase de suelo, relieve y textura (Tabla 4). Así, el mayor desarrollo de los árboles en el área AN1 puede ser una consecuencia de las diferencias de manejo entre las dos parcelas, desde el plantío hasta el momento actual. Es importante recordar que los años de 2014 a 2016 fueron extremamente atípicos en cuanto a la precipitación pluvial anual, con precipitaciones concentradas y debajo de la media histórica. Diversas plantaciones forestales fueron afectadas negativamente en la región, principalmente plantaciones nuevas. Estas plantaciones tuvieron perjuicios de stand (mortalidad) y crecimiento inicial (arranque).

Con todo, la progenie 15 presentó mayor divergencia en las dos áreas, seguida por la progenie nueve (Tabla 3). Esa diversidad puede estar relacionada con la segregación genética ocurrida durante la polinización abierta, el flujo génico entre los individuos y otros factores que proporcionan variación genética entre los individuos dentro de la población (Ashley, 2010). A pesar de que los individuos pertenecen a las mismas progenies, la contribución efectiva de la varianza aditiva, dominancia, epistática y epigenética son diferentes para cada evento reproductivo y llevan a variaciones dentro de cada progenie (Resende, 2007).

La distancia de Mahalanobis, en conjunto con el método de aglomeración de Tocher, es una de las metodologías más exactas para aglomerar por criterio de clasificación los individuos, genitores o progenies en grupos. La exactitud de ese método se debe al hecho de usar los valores genéticos predichos al contrario de fenotípicos a partir de las matrices de varianzas y covarianzas (Resende, 2007). Los grupos son formados de tal manera que tengan homogeneidad dentro del grupo y heterogeneidad entre grupos (Cruz et al., 2004, Bertan et al., 2006). Los valores genéticos predichos son usados en lugar de los valores fenotípicos, pues contribuyen al análisis de las matrices de varianzas y covarianzas de los valores genéticos que completan el componente de distancia de Mahalanobis, permitiendo así alta precisión en la formación de los grupos por el método de Tocher (Resende, 2007).

Con la finalidad de obtener gano genético en los ciclos subsecuentes de mejoramiento es recomendable la selección de progenies que se encuentren en grupos de mayor divergencia y que tengan mayor desempeño productivo (Ashley, 2010). De manera general, cruzamientos controlados entre individuos/progenies de grupos divergentes son indicados para aumentar la variabilidad genética (Resende, 2007). Para las progenies del área 1 (Tabla 3), deben realizarse cruzamientos entre los individuos del grupo 1 con la progenie del grupo 10, así sucesivamente para ganar diversidad genética. En el área 2, se recomienda que se haga el cruzamiento de las progenies del grupo 1 con la progenie del grupo 4 (Tabla 3). Esos cruzamientos y combinaciones son indicados para exploración de individuos intraespecíficos, en ciclos de mejoramiento, promoviendo el estudio detallado de los efectos de la capacidad de combinación especíifca sobre los caracteres estudiados. Nikles y Griffin (1992) relatan que la supremacía híbrida puede suceder a partir de la heterosis "per se", epistaxis o complementariedad de caracteres (efecto aditivo). Todos esos efectos podrán ocurrir entre individuos de grupos divergentes (Santos et al., 2016). 
TABLA 4. Algunas características físicas y químicas de puntos de observación en las parcelas del ensayo con louro-pardo y jacarandá (espaciamiento $4 \mathrm{~m} \times 4 \mathrm{~m}$ ) en tallones experimentales de la Reserva da Vale, Sooretama, ES.

\begin{tabular}{|c|c|c|c|c|c|c|c|c|c|c|}
\hline \multirow[b]{2}{*}{ Horizonte } & \multirow[t]{2}{*}{ Unidad } & \multicolumn{5}{|c|}{$\begin{array}{c}\text { AN1 - } 20 \text { m de altitud, UTM } 385699 \text { m Este y } \\
7883261 \text { m Sur, Arcsuelo Amarillo Distrocoeso } \\
\text { típico, horizonte A moderado, textura } \\
\text { média/arcilloso, topo amplio y plano, ensayo en } \\
\text { mejor condición }\end{array}$} & \multicolumn{4}{|c|}{$\begin{array}{c}\text { AN2 - } 24 \text { m de altitud, UTM } 386222 \text { m Este y } \\
7881663 \text { m Sur, Arcsuelo Amarillo Distrocoeso } \\
\text { típico, horizonte A moderado. Textura } \\
\text { média/arcillosa, topo amplio y plano, ensayo } \\
\text { en peor condición }\end{array}$} \\
\hline & & O & Ap & $B A$ & Btl & $\mathrm{Bt} 2$ & O & Ap & Btl & $\mathrm{Bt} 2$ \\
\hline Profundidad & $(\mathrm{cm})$ & $-1-0$ & $0-20$ & $20-35$ & $35-50$ & $50-85+$ & $-1-0$ & $0-20$ & $20-50$ & $50-100+$ \\
\hline $\begin{array}{l}\text { Arena } \\
\text { gruesa }\end{array}$ & & - & 65 & - & 40 & - & - & 65 & - & 40 \\
\hline Arena fina & $\begin{array}{l}\text { (dag } \\
\left.\mathrm{kg}^{-1}\right)\end{array}$ & - & 12 & - & 17 & - & - & 12 & - & 17 \\
\hline Limo & & - & 2 & - & 2 & - & - & 2 & - & 3 \\
\hline Arcilla & & - & 21 & - & 42 & - & - & 21 & - & 39 \\
\hline $\begin{array}{l}\text { Clase } \\
\text { textural }\end{array}$ & & - & $\begin{array}{l}\text { franco } \\
\text { arcillo } \\
\text { arenoso }\end{array}$ & - & $\begin{array}{l}\text { Arcillo } \\
\text { arenoso }\end{array}$ & - & - & $\begin{array}{l}\text { franco } \\
\text { arcillo } \\
\text { arenoso }\end{array}$ & - & $\begin{array}{c}\text { Arcillo } \\
\text { arenoso }\end{array}$ \\
\hline $\mathrm{CO}^{1}$ & $\begin{array}{l}(\mathrm{dag} \\
\left.\mathrm{dm}^{-3}\right)\end{array}$ & - & 0,75 & - & 0,23 & - & - & 0,69 & - & 0,20 \\
\hline$P$ & $\begin{array}{l}(\mathrm{mg} \\
\left.\mathrm{dm}^{-3}\right)\end{array}$ & - & 0,94 & - & 0,51 & - & - & 0,66 & - & 0,45 \\
\hline $\mathrm{pH} \mathrm{H} \mathrm{H}_{2} \mathrm{O}$ & & - & 5,20 & - & 6,20 & - & - & 6,00 & - & 5,00 \\
\hline $\mathrm{Ca}$ & & - & 1,26 & - & 1,07 & - & - & 1,24 & - & 0,52 \\
\hline $\mathrm{Mg}$ & & - & 0,72 & - & 0,29 & - & - & 0,46 & - & 0,18 \\
\hline K & & - & 0,10 & - & 0,04 & - & - & 0,07 & - & 0,07 \\
\hline Al & $\begin{array}{l}(\mathrm{cmolc} \\
\left.\mathrm{dm}^{-3}\right)\end{array}$ & - & 0,18 & - & 0,33 & - & - & 0,20 & - & 0,84 \\
\hline $\mathrm{H}+\mathrm{Al}$ & & - & 2,40 & - & 2,17 & - & - & 2,42 & - & 2,62 \\
\hline $\mathrm{SB}^{1}$ & & - & 2,08 & - & 1,40 & - & - & 1,77 & - & 0,77 \\
\hline СTC $^{1}$ & & - & 4,48 & - & 3,57 & - & - & 4,19 & - & 3,39 \\
\hline $\mathrm{V}^{1}$ & (\%) & - & 46 & - & 39 & - & - & 42 & - & 23 \\
\hline $\mathrm{ml}$ & & - & 8 & - & 19 & - & - & 10 & - & 52 \\
\hline $\begin{array}{l}\mathrm{Fe}_{2} \mathrm{O}_{3} \text { at. } \\
\text { sulfúrico }\end{array}$ & $\begin{array}{l}\text { (dag } \\
\left.\mathrm{kg}^{-1}\right)\end{array}$ & - & - & - & 2,79 & - & - & - & - & 2,72 \\
\hline $\mathrm{Ki}$ & & - & - & - & 1,86 & - & - & - & - & 1,72 \\
\hline
\end{tabular}

'CO = C orgánico, SB = suma de bases, CTC = capacidad de intercambio catiónico, $\mathrm{V}$ = saturación por bases, m = saturación por Al. 
Cuando el objetivo sea la recuperación de genes de los genitores (materno y paterno), el retrocruzamiento debe realizarse, preferentemente, con progenitores más similares genéticamente (del mismo grupo). El empleo de genitores similares, diferenciados básicamente por el gen a ser transferido, permite recuperar el genitor recurrente más rápidamente (Dias y Resende, 2001; Kumar et al., 2016).

Un próximo ciclo de mejoramiento genético de la especie para cualquier caracter de interés económico podrá ser realizado con cruzamientos de grupos divergentes. Esos nuevos individuos que conformarán el próximo ciclo podrán ser superiores a la media de los parentales, sea a partir del efecto de heterosis per se, complementariedad o heterosis funcional (Li, Howe y Wu, 1998; Lamkey, 1999). Tanto la heterosis como la capacidad específica de combinación (CEC) entre dos pares genitores divergentes dependen de la existencia de la dominancia en el control del caracter y de la presencia de divergencia (Falconer y Mackay, 1996). Para los grupos de genotipos más divergentes existe la posibilidad de evaluarse la capacidad general y específica de combinación de esas matrices, así como explorar la heterosis con base en los efectos aditivos y dominancias, con enorme posibilidad de progenies más productivas que sus parentales (Santos et al., 2016; Kumar et al., 2016).

\section{CONCLUSIONES}

Las progenies de Cordia trichotoma presentan alta divergencia genética en las dos áreas y los caracteres que más contribuyeron para divergencia genética fueron el DAP2016 y ALT-2014 en el área 2, por tanto, esos caracteres deben ser considerados y priorizados en los cruzamientos para exploración de la heterosis.

Por el método de agrupamiento de Tocher, las progenies fueron separadas en 10 grupos en el área 1 y cuatro grupos en el área 2. Para programas de mejoramiento, se recomienda el cruzamiento entre progenies de grupos más divergentes para priorizar el gano con selección con la exploración de cruzamientos de individuos con mayor potencial productivo.

\section{RECONOCIMIENTOS}

Los autores agradecen a la Coordenação de Aperfeiçoamento de Pessoal de Nível Superior (CAPES). Empresa Vale. Empresa de Investigación Embrapa Foresta, y a los técnicos investigadores del Proyecto Biomas, Embrapa Foresta y FEIS - UNESP (Universidade Estadual Paulista em Ilha Solteira).

\section{REFERENCIAS}

Alves R., M., García A., A., F., Cruz E., D., \& Figueira, A. (2003). Seleção de descritores botânico-agronômicos para caracterização de germoplasma de cupuaçuzeiro. Pesquisa Agropecuária Brasileira, 38(7), 807-818. doi: 10.1590/S0100-204X2003000700004

Alvares, C., A., Stape, J., L., Sentelhas, P., C., Gonçalves, J., L., M. \& Sparovek, G. (2013). Köppen's climate classification map for Brazil. Meteorologische Zeitschrift 22, 711-728.

Ashley M., V. (2010). Plant parentage, pollination, and dispersal: How DNA microsatellites have altered the landscape. Critical Reviews in Plant Sciences, 29(1), 148-161. doi: $10.1080 / 07352689.2010 .481167$

Bertan, I., Carvalho F., I., F., Oliveira A., C., Vieira E., A., Hartwig, I., Silva J., A., G., Shimidt D., A., M., Valério I., P., Busato C., C., \& Ribeiro, G. (2006). Comparação de métodos de agrupamento na representação da distância morfológica entre genótipos de trigo. Revista Brasileira de Agrociência, 12(3), 279-286. doi: 10.18539/CAST.V12I3.455

Coser S., M., Motoike S., Y., Corrêa T., R., Pires T., P., \& Resende M., D., V. (2016). Breeding of Acrocomiaaculeata using genetic diversity parameters and correlations to select accessions based on vegetative, phenological, and reproductive characteristics. Genetics and molecular research, 15(4), 2-11. doi: 0.4238/gmr15048820

Costa R., B., Silva J., C., Skowronski, L., Constantino, M., Pistori, H., \& Pinto J., V., C. (2016). Genetic Divergence in Eucalyptus camaldulensis Progenies in the Savanna Biome in Mato Grosso, Brazil. Plos One, 11(9), 1-9. doi: 10.1371/journal.pone.0163698

Cruz C., D., \& Regazzi A., J. (2001). Modelos biométricos aplicados ao melhoramento genético ( $1^{\mathrm{a}}$ ed.). Viçosa, Brasil: Universidade Federal de Viçosa.

Cruz C., D., \& Carneiro P., C., S. (2003). Modelos biométricos aplicados ao melhoramento genético ( $2^{\mathrm{a}}$ ed.). Viçosa, Brasil: Universidade Federal de Viçosa.

Cruz C., D., Regazzi A., J., \& Carneiro P., C., S. (2004). Modelos biométricos aplicadas ao melhoramento genético ( $3^{\mathrm{a}}$ ed.). Viçosa, Brasil: Universidade Federal de Viçosa. 
Cruz C., D. (2006). Programa Genes: análise multivariada e simulação (1ª ed.). Viçosa, Brasil: Universidade Federal de Viçosa.

Correa A., M., \& Gonçalves M., C. (2012). Divergência genética em genótipos de feijão comum cultivados em Mato Grosso do Sul. Revista Ceres, 59(2), 206-212. doi: 10.1590/S0034737X2012000200009.

Cui, Z., Thomas, E., Carter J., R., Joseph W., B., Randy, W. (2001). Phenotypic diversity of modern Chinese and North American soybean cultivars. Crop Science, 41(6), 1954-1967. doi: 10.2135/cropsci2001.1954

Dias, L. A. S., \& Resende, M. D. V. (2001). Estratégias e métodos de seleção. En L. A. S. Dias (Eds.), Melhoramento genético do cacaueiro (pp. 1-39). Viçosa, Brasil: Universidade Federal de Viçosa.

Falconer D., S., \& Mackay T., F., C. (1996). Introduction to quantitative genetics ( $4^{\mathrm{a}}$ ed.). Harlow, Inglaterra: Longman.

Kumar, A., Ravichandran, S., Dobhal, S., \& Kumar V., D. (2016). D2 Analysis for estimating genetic divergence in different clones of Dalbergia sissoo. Journal of Forestry Research, 27(5), 1085-1097. doi: 10.1007/s11676-016-0224-3

Instituto Capixaba de Pesquisa, Assistência Técnica e Extensão Rural [Incaper] (2013). Programa de assistência técnica e extensão rural proater (2013). Recuperado por: http://www.incaper.es.gov.br/proater/municipios/Nordeste/S ooretama.pdf

Lamkey, K. R. (1999). Quantitative genetics of heterosis. En Coors, J. G. Pandey, S. (Eds), Genetics and exploitation of heterosis in crops (pp. 3143). Madison: American Society of Agronomy.

Li, B., Howe, G., T., \& Wu, R. (1998). Developmental factors responsible for heterosis in aspen hybrids (Populustremuloides $\times P$. tremula). Tree Physiology, 18(1), 29-36. doi: 10.1093/treephys/18.1.29

Ministério do Meio Ambiente [MMA]. (2011). Espécies nativas da flora brasileira de valor econômico atual ou potencial: plantas para o futuro Região Sul. Recuperado de: https://www.mma.gov.br/estruturas/sbf2008_dcbio/_ebooks/ regiao_sul/Regiao_Sul.pdf

Negreiros, J., R., S., Bergo, C., L., Migueloni, D., P., \& Pereira, A., M. (2013). Divergência genética entre progênies de pupunheira quanto a caracteres de palmito. Pesquisa agropecuária brasileira, 48(5), 496-503. doi: 10.1590/S0100-204X2013000500005
Nikles D., G., \& Griffin A., R. (1992). Breeding hybrids of forest trees: definitions, theory, some practical examples, and guidelines on strategy with tropical acacias. Aciar Proceedings, 1(37), 101-109.

Resende, M., D., V. (2007). Matemática e estatística na análise de experimento e no melhoramento genético ( $\left.1^{\mathrm{a}} \mathrm{ed}\right)$. Colombo, Brasil: Centro Nacional de Pesquisas Florestais.

Resende, M., D., V. (2016) Software Selegen-REML/BLUP: a useful tool for plant breeding. Crop Breeding and Applied Biotechnology, 16(4), 330-339. doi: 10.1590/1984-70332016v16n4a49

Santos, W., Araújo, E., G., Souza, D., C., L., Silva, J., R., Recco, C., R., W., B., Moraes, M., L., T., \& Aguiar, A., V. (2016). Divergência genética entre progênies de polinização aberta de Pinus caribaea var. hondurensis a partir de caracteres quantitativos. Pesquisa Florestal Brasileira, 36(86), 127-133. doi: 10.4336/2016.pfb.36.86.920

Santos, W., Souza, B., M., Zulian, D., F., Alves, G., T., R., Moraes, M., L., T., Sousa, V., A., Aguiar, A., V. (2020 Não Publicado, It was accepted on July 29, 2020.). Environment-genotype interaction in Cordia trichotoma (Vell.) Arráb. Ex Steud. Progenies in two different soil conditions. Journal of Forestry Research

Singh, D. (1981). The relative importance of characters affecting genetic divergence. The Indian Journal of Genetics e Plant Breeding, 41(2), 237 245.

Manuscrito recibido el 16 de junio de 2020

Aceptado el 14 de enero de 2021

Publicado el 30 de diciembre de 2021

\section{Este documento se debe citar como:}

Santos, W. dos, Souza, B. M. de, Zulian, D. F., Alves, G. T. R., Moraes, M. L. T. de, Sousa, V. A. de, Aguiar, A. V. de (2021). Divergencia genética entre progenies de Cordia trichotoma en una plantación mixta con Dalbergia nigra. Madera y Bosques, 27(2), e2722188. doi: 10.21829/myb.2021.2722188 